Corresponding authors: Elaine. Mardis@nationwidechildrens. org; Rubin_J@wustl.edu

(C) 2018 Miller et al. This article is distributed under the terms of the Creative Commons Attribution License, which permits unrestricted reuse and redistribution provided that the original author and source are credited.

Ontology terms: ependymoma; neoplasm of the central nervous system; neoplasm of the nervous system

Published by Cold Spring Harbor Laboratory Press

doi: $10.1101 / \mathrm{mcs} . \mathrm{a} 002444$

\section{Resistance-promoting effects of ependymoma treatment revealed through genomic analysis of multiple recurrences in a single patient}

\author{
Christopher A. Miller, ${ }^{1,2}$ Sonika Dahiya, ${ }^{3}$ Tiandao Li, ${ }^{2}$ Robert S. Fulton, ${ }^{2}$ \\ Matthew D. Smyth, ${ }^{4}$ Gavin P. Dunn, ${ }^{4}$ Joshua B. Rubin, ${ }^{5}$ and Elaine R. Mardis ${ }^{6}$ \\ ${ }^{1}$ Department of Medicine, Division of Oncology, Washington University School of Medicine, St. Louis, \\ Missouri 63110, USA; ${ }^{2}$ McDonnell Genome Institute, Washington University School of Medicine, St. Louis, \\ Missouri 63110, USA; ${ }^{3}$ Department of Pathology and Immunology, Washington University School of Medicine, \\ St. Louis, Missouri 63110, USA; ${ }^{4}$ Department of Neurological Surgery, Washington University School of \\ Medicine, St. Louis, Missouri 63110, USA; ${ }^{5}$ Department of Pediatrics, Washington University School of \\ Medicine, St. Louis, Missouri 63110, USA; ${ }^{\circ}$ Institute for Genomic Medicine, Nationwide Children's Hospital, \\ and The Ohio State University College of Medicine, Columbus, Ohio 43205, USA
}

\begin{abstract}
As in other brain tumors, multiple recurrences after complete resection and irradiation of supratentorial ependymoma are common and frequently result in patient death. This standard-of-care treatment was established in the pregenomic era without the ability to evaluate the effect that mutagenic therapies may exert on tumor evolution and in promoting resistance, recurrence, and death. We seized a rare opportunity to characterize treatment effects and the evolution of a single patient's ependymoma across four recurrences after different therapies. A combination of high-depth whole-genome and exomebased DNA sequencing of germline and tumor specimens, RNA sequencing of tumor specimens, and advanced computational analyses were used. Treatment with radiation and chemotherapies resulted in a substantial increase in mutational burden and diversification of the tumor subclonal architecture without eradication of the founding clone. Notable somatic alterations included a MEN1 driver, several epigenetic modifiers, and therapy-induced mutations that impacted multiple other cancer-relevant pathways and altered the neoantigen landscape. These genomic data provided new mechanistic insights into the genesis of ependymoma and pathways of resistance. They also revealed that radiation and chemotherapy were significant forces in shaping the increased subclonal complexity of each tumor recurrence while also failing to eradicate the founding clone. This raises the question of whether standard-of-care treatments have similar consequences in other patients with ependymoma and other types of brain tumors. If so, the perspective obtained by real-time genomic characterization of a tumor may be essential for making effective patientspecific and adaptive clinical decisions.
\end{abstract}

[Supplemental material is available for this article.]

\section{INTRODUCTION}

Ependymomas are a heterogeneous group of primary central nervous system (CNS) tumors with multiple histological, brain region, age, and molecular features distinguishing between different prognostic groups (Pajtler et al. 2015; Dorfer et al. 2016; Khatua et al. 2017). Based 
COLD SPRING HARBOR Molecular Case Studies
Treatment-linked resistance in an ependymoma on standard histological features, ependymal neoplasms can be diagnosed as World Health Organization (WHO) Grade I, II, or III tumors. However, in contrast to other brain tumors, histological grading has proven to be a weak prognostic indicator of outcome for ependymomas (Pajtler et al. 2017). In the largest published study of ependymoma outcome involving 282 patients, gross total resection (GTR) was the only prognostic factor associated with increased survival (Vera-Bolanos et al. 2015). Strikingly, in this study, GTR and postsurgical radiation therapy associated with a shorter progression-free survival than GTR alone. These data indicate that, as yet, we do not know enough about the molecular mechanisms of ependymoma, or about the appropriate indications for, and most effective modes of, adjuvant therapies.

A means to generating the necessary insights to address these concerns is comparative genomic analyses of primary and posttreatment specimens. To date, there is a paucity of information regarding the genomic changes in ependymomas that recur serially through multiple treatment regimens. This is largely due to the rarity of the disease and a failure to consistently bank and analyze recurrent samples. To determine the temporal genomic changes that occurred in one patient's ependymoma disease as it recurred after several different therapeutic modalities, we characterized the genomic landscape of serial resections with high-depth whole-genome and exome sequencing. These data provided an evaluation of putative driver mutations, mutational signatures resulting from therapy, mechanisms for therapy response and resistance, and shifts in the neoantigen profile from the initial disease presentation through four recurrences.

\section{CLINICAL PRESENTATION AND FAMILY HISTORY}

The initial diagnosis was made in a 16-yr-old right-handed female who presented to the St. Louis Children's Hospital Emergency Department with a 3-d history of headache and vomiting (Table 1). Magnetic resonance imaging (MRI) scan revealed a $6 \times 4 \mathrm{~cm}$ enhancing mass in the right frontotemporal region (Fig. 1A, initial diagnosis). The patient underwent a GTR via a right frontotemporal craniotomy. Pathological evaluation was significant for a hypercellular glial tumor with prominent pseudo-rosettes, increased mitoses, vascular

\begin{tabular}{ll}
\hline Table 1. Clinical history & \\
\hline Date & \multicolumn{1}{c}{ Event } \\
\hline Month 1 & Initial GTR of anaplastic ependymoma WHO Grade III \\
Months 2-3 & Irradiation of tumor bed to 59.4 Gy \\
Month 45 & GTR of first recurrent anaplastic ependymoma \\
Months 47-48 & Reirradiation with 59.4 Gy with 10 months of temozolomide \\
Months 48-58 & Temozolomide chemotherapy \\
Month 63 & GTR of second recurrent anaplastic ependymoma \\
Month 65 & Lapatinib and Avastin therapy initiated \\
Month 69 & Lapatinib discontinued secondary to toxicity \\
Month 77 & Avastin discontinued \\
Month 83 & GTR of third recurrence of anaplastic ependymoma \\
Months 84-104 & Avastin therapy \\
Month 104 & GTR of fourth recurrent anaplastic ependymoma
\end{tabular}

GTR, gross total resection; WHO, World Health Organization. 
COLD SPRING HARBOR Molecular Case Studies
Treatment-linked resistance in an ependymoma
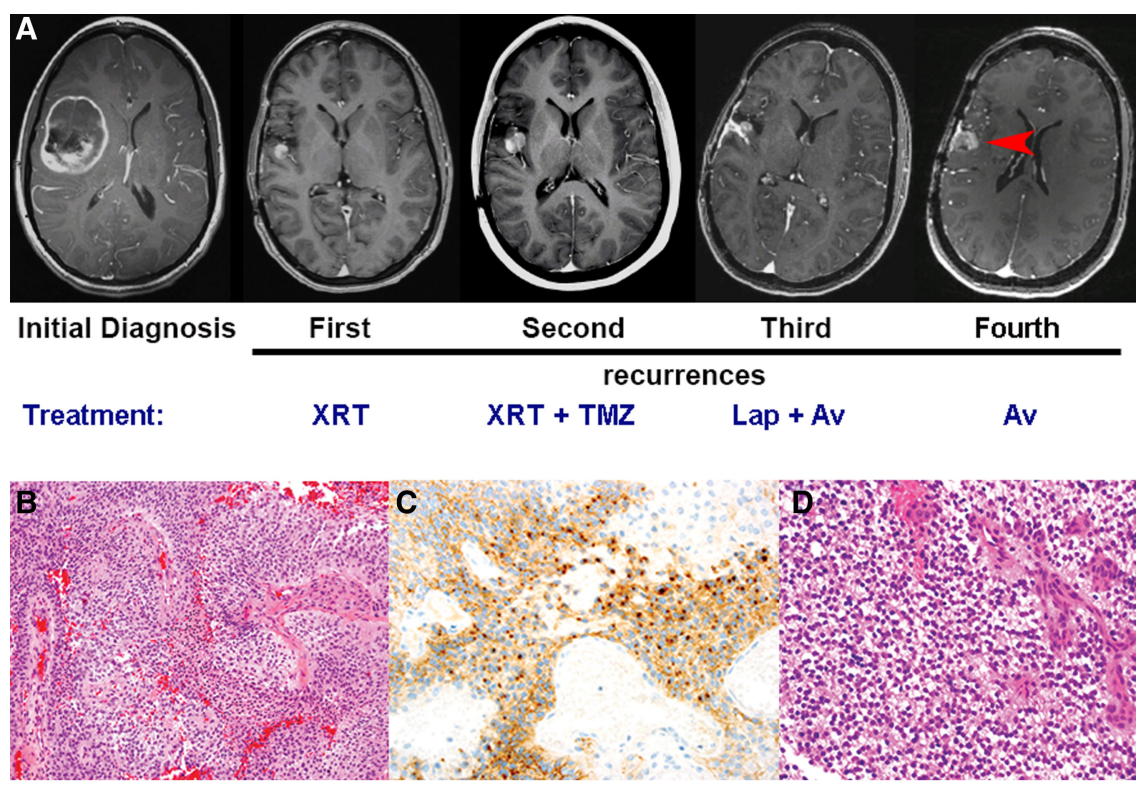

Figure 1. Radiographic and pathological evaluation of initial and recurrent ependymoma. (A) Serial MRIs over a 9-yr period demonstrating a heterogeneously enhancing mass in the right frontotemporal region at the time of initial diagnosis and four enhancing recurrent lesions adjacent to the initial resection cavity. (B) Hematoxylin and eosin (H\&E) stain of formalin-fixed paraffin-embedded primary resection material revealed a densely cellular tumor with increased mitotic activity, necrosis, and microvascular proliferation. (C) Immunostain for epithelial membrane antigen (EMA) shows multifocal perinuclear dot-like positivity, which is characteristic of ependymal differentiation along with concomitant cytoplasmic expression of glial fibrillary acidic protein (not shown). (D) H\&E stain of each recurrent tumor revealed persistence of the ependymal phenotype. Pictured is the third recurrence. All the photomicrographs are taken at $40 \times$ magnification.

proliferation and necrosis, and perinuclear dot-like expression of epithelial membrane antigen (EMA) (Fig. 1B,C) along with diffuse glial fibrillary acidic protein immunoreactivity. A diagnosis of anaplastic ependymoma (WHO Grade III) was made. Evaluations for CNS dissemination were negative. The patient received 59.4 Gy of fractionated photon irradiation to the tumor bed plus a $1-\mathrm{cm}$ margin, which is standard for supratentorial ependymoma. Forty-four months after the initial diagnosis, the patient suffered a seizure and an MRI revealed a $13 \times 15 \times 16 \mathrm{~mm}$ nodular recurrence in the right frontal lobe along the posterior margin of the initial resection cavity (Fig. 1A, first recurrence). MRI of spine and cerebrospinal fluid cytology were negative. The patient underwent complete resection of the recurrent tumor, which exhibited similar histology to the initial tumor. The resection cavity and margin were reirradiated with an additional 59.4 Gy of fractionated photon irradiation and the patient received 10 mo of standard dose temozolomide treatment.

A surveillance scan 17 mo after the second resection demonstrated a 7-mm enhancing nodule in the temporal surface of the right sylvian fissure near the resection cavity, consistent with recurrence (Fig. 1A, second recurrence). Following a third complete resection, histopathology was again consistent with anaplastic ependymoma and analysis for dissemination was negative. The patient was enrolled on CERN-0801 at Children's Memorial Hospital in Chicago and received combined Avastin and lapatinib. Lapatinib was discontinued 4 mo later because of toxicity, and Avastin was continued for an additional 8 mo for a total of $1 \mathrm{yr}$ of treatment every $2 \mathrm{wk}$. Six months later, an MRI revealed a new right perisylvian lesion and right thalamic enhancing nodule (Fig. 1A, third recurrence). Complete resection of the perisylvian lesion was performed and pathology again indicated anaplastic ependymoma (Fig. 1D) with no evidence of dissemination. Avastin was restarted and continued for 20 mo until 
new evidence from serial MRI indicated progression in a perisylvian lesion that had remained following the most recent surgery (Fig. 1A, fourth recurrence). This lesion also was completely resected and diagnosed as anaplastic ependymoma. The patient continues on treatment at the time of this report, $>11 \mathrm{yr}$ from original diagnosis, without evidence of dissemination beyond this loco-regional area.

\section{GENOMIC ANALYSES}

\section{Analysis of the Matched Normal Sample}

To determine whether the patient possessed a germline predisposition to cancer, we analyzed the sequence data obtained from her leukocyte-derived DNA (normal, Supplemental Table S1) and identified 176 protein-altering constitutional variants that were rare in the population and fell into highly damaging classes of mutations (frameshifts, nonsense, nonstop, or splice-site). Variants were observed in several genes known to be important for immune function, including splice site SNPs in RAG1, HLA-DRB1, and HLADRB5, as well as a nonsense mutation in HLA-DRB5. Several cancer-relevant genes were also observed: splice-site alterations in DDX3X (Dahlin et al. 2015) and MAD2L2 (alias: REV7) (Boersma et al. 2015; Xu et al. 2015) and in-frame insertions in MNX1 (Das 2016) and ZFHX3 (Mabuchi et al. 2010). Some with direct glioma relevance were also observed: FOXD1 (in-frame deletion) (Koga et al. 2014; Cheng et al. 2016; Gao et al. 2017), BCL2L2 (SNP) (Chung et al. 2015), and RYK (frameshift insertion) (Adamo et al. 2017). Although MNX1 functions as an oncogene to promote pancreatic islet cell tumors in multiple endocrine neoplasia type 1 (MEN1) (Scacheri et al. 2006), this particular mutation is common in the population and unlikely to be relevant to predisposition.

\section{Landscape of Somatic Mutations during Disease Progression}

We identified 1332 somatic mutations across the five resection specimens, 162 of which were in protein-coding regions, and 110 of which were nonsilent (Fig. 2; Supplemental Table S2). The primary tumor sample contained only one overtly cancer-related gene mutation, an expressed frameshift insertion in MEN1 (K237fs) (Table 2). We also observed several large copy-number alterations (CNAs) in this sample, including deletions of 6p, 15q, 22, and the first $22 \mathrm{Mb}$ of Chromosome 1, that were shared with the recurrent tumors (Supplemental Fig. S1, Table S3). Chromosome 11 was heavily rearranged, with multiple distinct regions of amplification and deletion, one of which deleted the second copy of MEN1. Integrated analysis of the DNA and RNA did not detect any gene fusion events, although many putative structural variants were detected (Supplemental Tables S4 and S5).

All SNVs, indels, and CNAs found in the initial resection were retained in the first recurrence, which was diagnosed after radiation therapy and a 44-mo interval. An additional 12 new protein-coding somatic mutations were identified in the recurrent tumor, including a nonsense mutation in DEPDC5, an inhibitor of mTORC signaling. Missense mutations were observed in KREMEN2 (G165V), a gene that has been linked to melanoma, and in BANP (N223S), an epigenetic regulator. None is obviously expressed in this tumor, but the variants in both KREMEN2 and BANP are expressed in subsequent tumors with higher quality and higher-depth RNA-seq, so it is likely that these variants are expressed below our level of sensitivity in this resection sample. Mutated DEPDC5 may have been undetectable because of undergoing nonsense-mediated decay.

The second recurrent tumor emerged after additional radiation therapy and treatment with temozolomide. It was resected and the genomic analysis of this specimen indicated that essentially all previously observed mutations were retained, with the exception of 


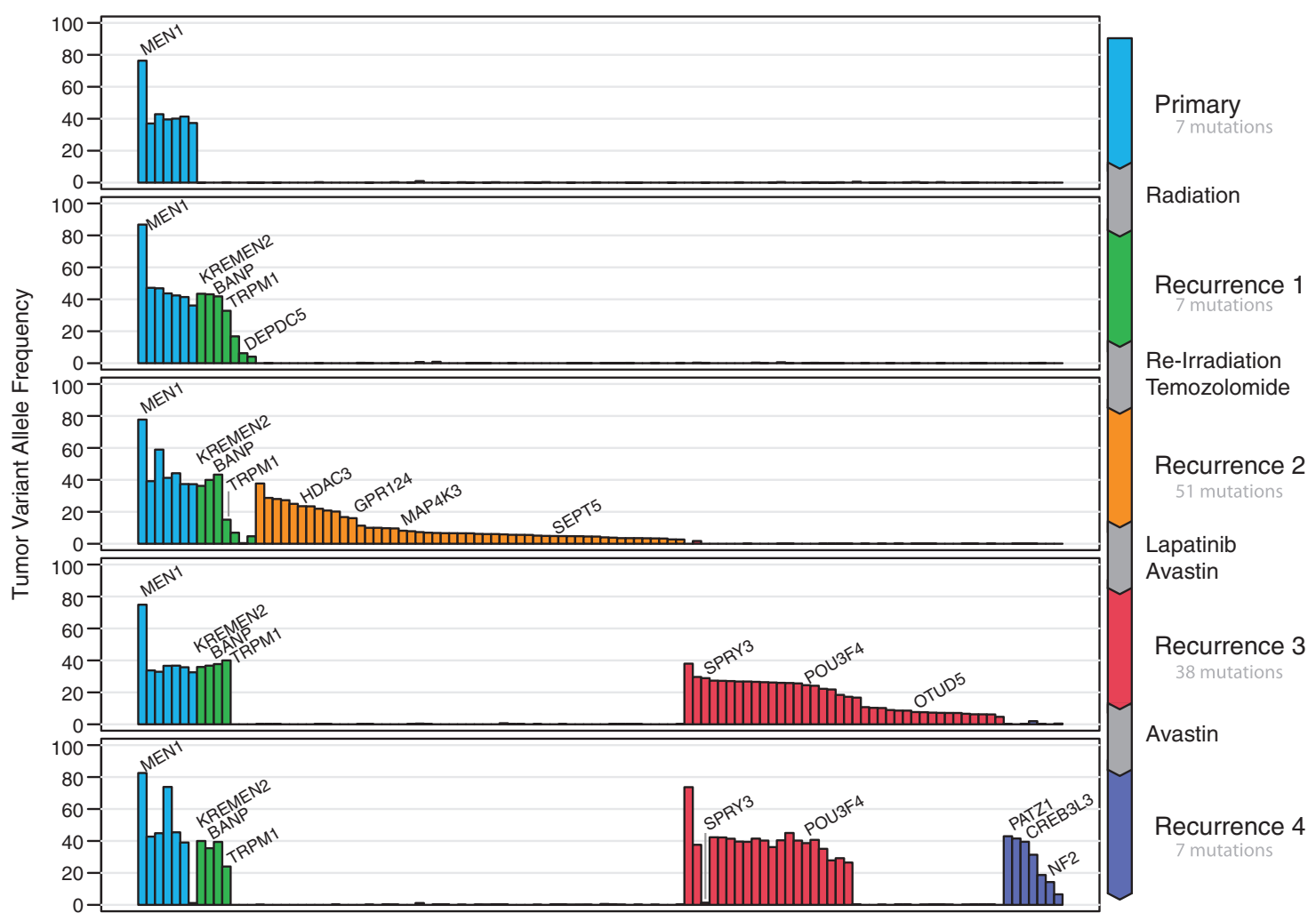

Figure 2. Variant allele fractions of nonsilent mutations in protein-coding genes in all five resections.

two low-VAF protein-coding variants from the previous recurrence, including loss of the DEPDC5 nonsense mutation. An additional 66 protein-coding SNVs and indels were acquired, including a 19-bp frameshift deletion in GPR124 and low-VAF missense mutations in SEPT5 (T260A), MAP4K3 (F300S), and KAT6B (P1276L). Of these, only the MAP43K and KAT6B mutations were observably expressed. The copy-number landscape was identical to the previous tumors, with the exception of a new homozygous deletion on Chromosome 2p.

The third recurrence occurred after treatment with Avastin and lapatinib. Genomic analysis of this resection specimen revealed that all coding mutations specific to the second recurrence, including the Chromosome 2 copy-number loss, were undetectable at the third recurrence. In contrast, virtually all mutations identified in the first two resections persisted, the only exception being two low-VAF events in MYH10 and OR1L1. Fifty-six new proteincoding mutations were acquired, including missense mutations in POU3F4 (P568T), an epigenetic regulator, OTUD5 (P338L), a p53 activator, and SPRY3 (R19C), a regulator of FGF signaling. None has been previously implicated in ependymoma, and their relevance for disease progression and therapy resistance is unclear.

The fourth recurrence was resected after continued Avastin treatment. In this sample, 29 of the protein-coding mutations newly acquired in the prior (third) recurrence were no longer detected, but 18 new protein-coding mutations were identified. These included nonsense mutations in CREB3L3 and NF2, a gene previously linked to ependymoma. A missense mutation in the chromatin/transcriptional regulator PATZ1 was also observed. In addition to the NF2 mutation, we identified two point mutations that potentially impact Hippo pathway signaling in LATS1 and MAP4K3 (Meng et al. 2015; Oh et al. 2015). 


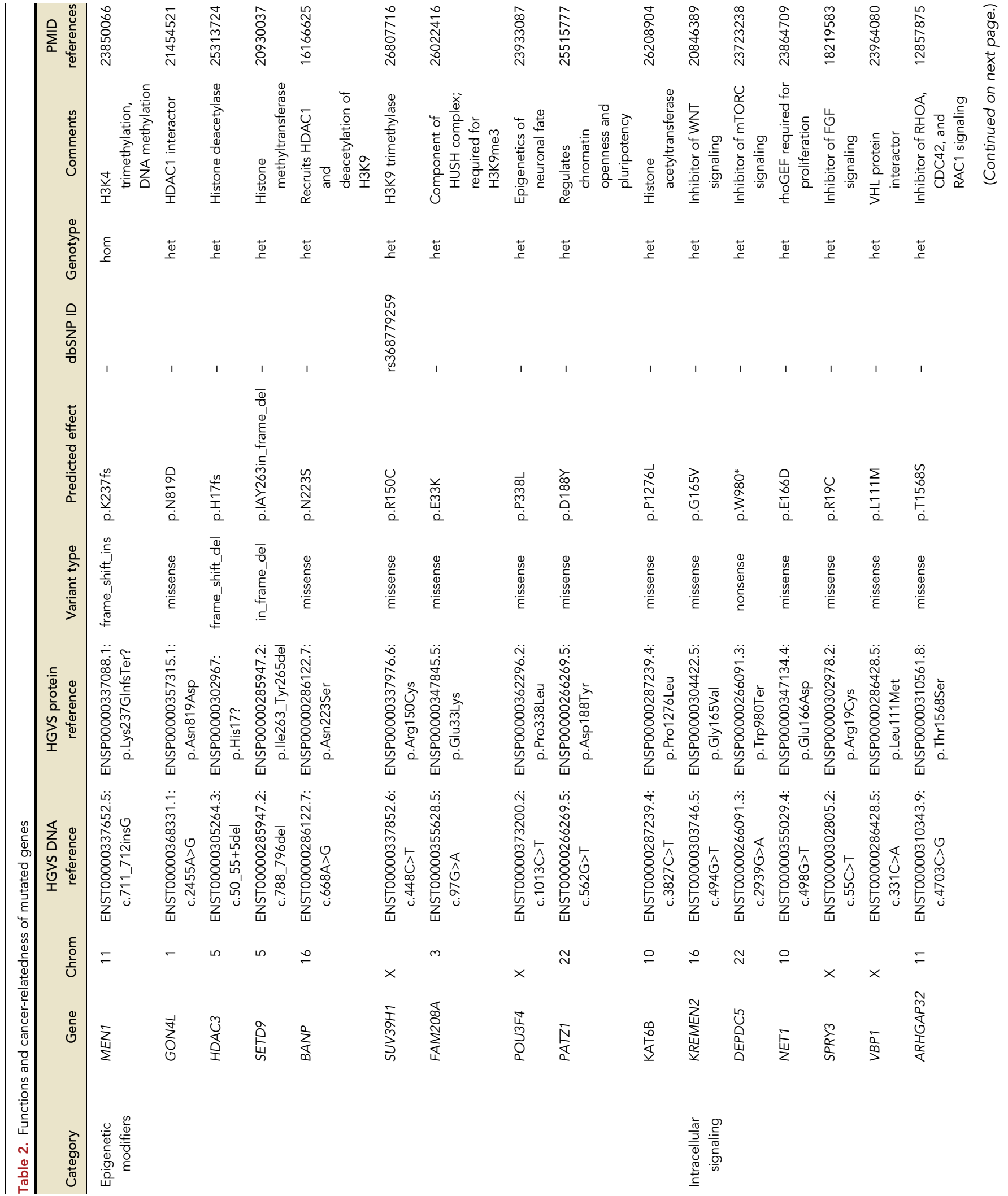




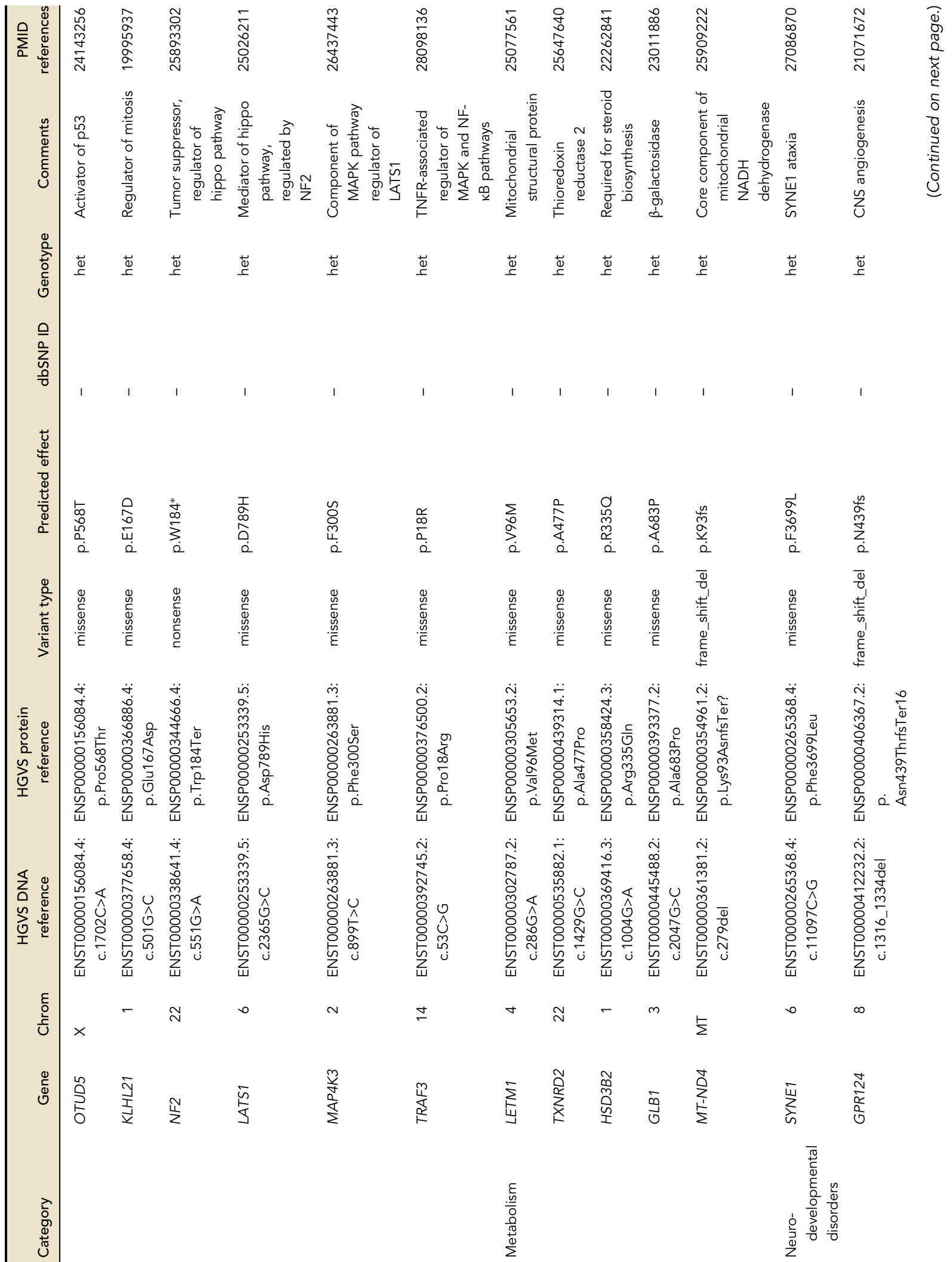




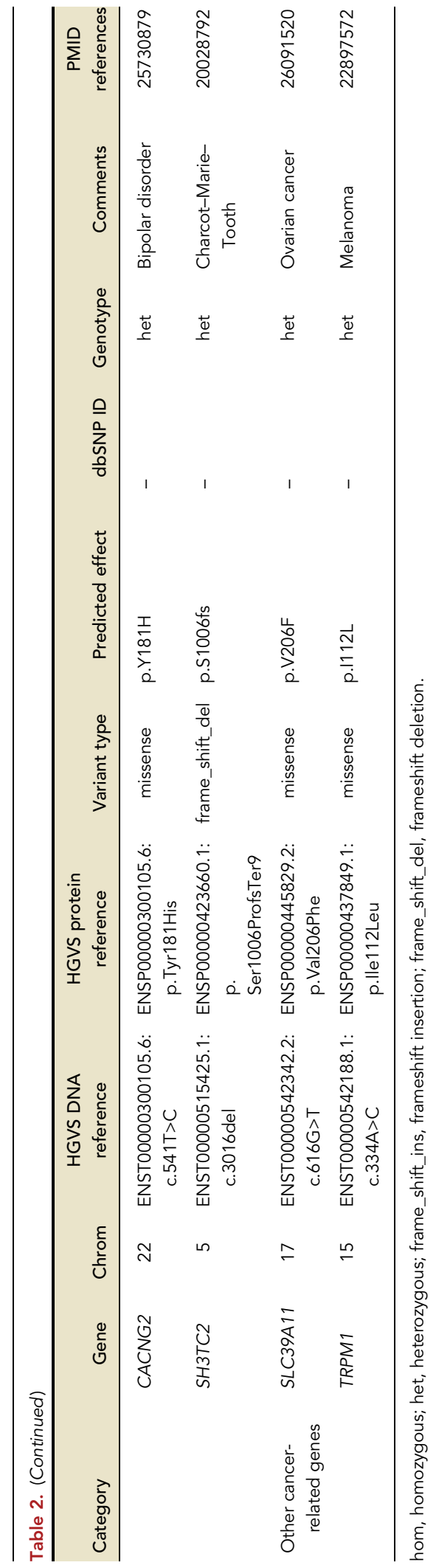




\section{Clonal Heterogeneity and Mechanisms of Tumor Evolution}

To characterize the changing clonal architecture of this tumor, the variant allele fractions of copy-number neutral SNVs were clustered in five dimensions using the sciClone algorithm (Fig. 3A). Eight clusters were detected, and the mutation spectrum for each was identified. The first recurrent tumor after radiation therapy was dominated by cluster 2, which emerged from a population of cells undetectable in our analysis of the original biopsy data (with a sensitivity of $2 \%$ VAF). The mutation spectrum shows a notable decrease in $\mathrm{C}>\mathrm{T}$ transitions in cluster 2, when compared with those in cluster 1 from the original tumor (Fig. 3B).

In the second recurrence, following additional radiation and treatment with temozolomide, we identified the emergence of two new subclonal populations (clusters 3 and 4) that were likewise undetectable in the prior two samples' data. Cluster 4, and all subsequently appearing clusters, each have a significantly higher proportion of $A>G$ transitions than the founding clone, a pattern consistent with temozolomide-induced mutagenesis (all $P<0.03$ ) (Supplemental Table S6; Bodell et al. 2003). In the third recurrence, following Avastin treatment, both clusters 3 and 4 were undetectable, but clusters 5 and 6 emerged. Although cluster 6 was cleared in the final resection sample we studied, cluster 5 persisted and two rare subclonal populations expanded into clusters 7 and 8 , which make up a

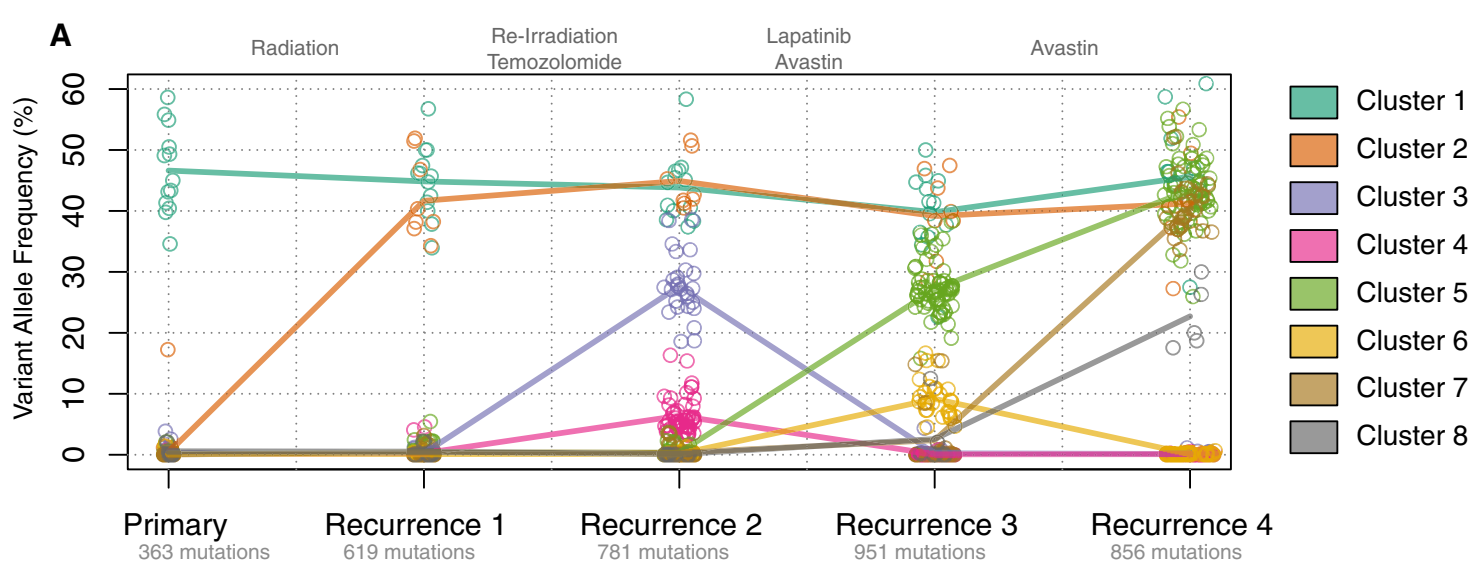

B

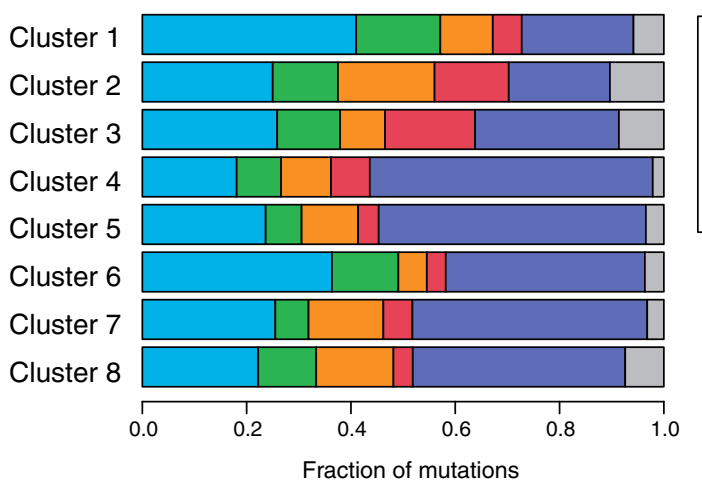

C

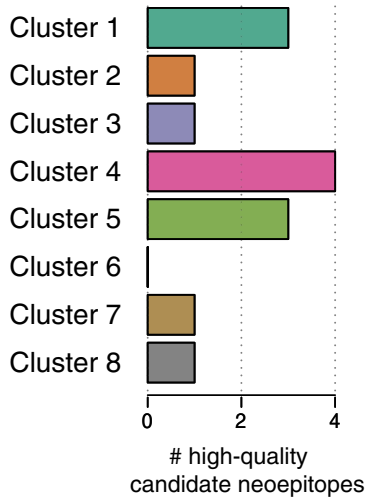

Figure 3. (A) Subclonal clustering of the five tumor samples. Points represent the VAFs of individual SNVs at each time point, and lines connect the mean VAF of each cluster in each sample. Each sample is labeled with the number of detectable mutations at that time point. (B) Mutation spectrum of each cluster. $(C)$ The number of high-quality MHC Class I neoantigens found in each subclonal population. 
substantial portion of the final tumor. Some mutations in these two clusters were just above the level of detection in the third resection.

Though there were too few mutations to perform per-cluster mutational signature analysis (Rosenthal et al. 2016), we did compare the deletion/substitution ratio between mutations that predated irradiation (cluster 1 ) and those that arose after radiotherapy (clusters 2-8). We observed a significant increase in the proportion of deletions postirradiation (Pearson's $\chi^{2} P=1.657 \times 10^{-05}$ ), a finding that is consistent with previously reported mutational signatures of ionizing radiation (Behjati et al. 2016).

In addition to identifying mutations correlated with specific subclonal expansions, we also examined the expression of $\mathrm{O}^{6}$-methylguanine DNA methyltransferase (MGMT), which is known to drive brain tumor recurrence through increased expression in post-temozolomide lesions (Bocangel et al. 2002; Hegi et al. 2005). In this case, MGMT RNA expression levels were not clearly correlated with the emergence of post-temozolomide recurrences, suggesting that they relied upon alternative mechanisms of resistance (Supplemental Fig. S2).

\section{Evolving Landscape of Targets for Immunotherapy}

To understand how the immunogenicity of this tumor evolved over the course of treatment, we applied the pVACSeq neoantigen prediction pipeline (Hundal et al. 2016) to the proteinaltering mutations that we observed in each tumor studied. The patient's HLA haplotypes were inferred to be $A^{*} 24: 02, A^{*} 26: 01, B^{*} 40: 02, B^{*} 38: 01, C^{*} 12: 03$, and $C^{*} 03: 05$. We identified only 14 expressed mutations that produce "high-quality" predicted MHC Class I neoantigens (Fig. 2C), which we define as having median binding affinity (ic50) of $<500 \mathrm{nM}$, and with a higher binding affinity to the mutant than the wild-type peptide (Supplemental Table S7). As overall mutation burden is highly correlated with neoantigen load, this is perhaps unsurprising. Only three neoantigens were present in the founding clone, whereas 11 of the 14 were specific to a subclonal population and therefore not present in all cells of the tumor.

\section{DISCUSSION}

In this study, we found that standard-of-care and experimental approaches to treatment of an ependymoma increased its mutational burden and diversified its subclonal architecture. The diagnosis of a supratentorial anaplastic ependymoma, not arising from the ventricles, is uncommon. Ependymomas, which occur in both pediatric and adult patients, display age-dependent patterns of location and histology. Overall, the majority of ependymomas occur in the spine, though this location is involved in only $20 \%$ of pediatric cases (McGuire et al. 2009a, 2009b). As was evident in this case, supratentorial ependymomas are most common in older children and adolescents. In addition, anaplastic histology is also more common in pediatric cases. Recent genomic analyses have indicated that most supratentorial anaplastic ependymomas are associated with fusion events involving RELA or YAP1 (Pajtler et al. 2015). Neither of these fusion events was detected in this case.

\section{The Role of MEN1 Mutations}

Notable in the evaluation of the primary diagnostic specimen was the biallelic somatic disruption of MEN1. MEN1 mutations, typically biallelic, have been infrequently reported in ependymoma, both in the context of familial MEN1 syndrome and sporadically (Kato et al. 1996; Urioste et al. 2002; Al-Salameh et al. 2010; Lee et al. 2016b), and appear to occur in both high- and low-grade tumors of any location. Although MEN1 mutation has been associated with recurrence and progression from grade II to III ependymoma (Urioste et al. 
2002; Funayama et al. 2013), we offer the first clear evidence that MEN1 mutation is a driver of the founding clone.

Functionally, MEN1 mutations are known to increase DNMT1 activity, which leads to global increases in CpG island methylation, a characteristic of other tumors (Funayama et al. 2013; Mack et al. 2014; Yuan et al. 2016), and silences key tumor suppressor genes, including CDKN1B, CDKN2A, APC, and RASSF1A (Karnik et al. 2005; Lindberg et al. 2008; Juhlin et al. 2010). Coupled with the observed mutations in other epigenetic regulators, these data add new evidence for epigenetic dysregulation in the genesis and progression of ependymoma.

Loss of menin function also leads to activation of the RAS (Wu et al. 2012), MAP kinase (Gallo et al. 2002), PI3 kinase (Wang et al. 2011), Sonic Hedgehog (Gurung et al. 2013), Wnt (Cao et al. 2009), and TGF- $\beta$ signaling pathways, all with established roles in gliomagenesis (Matkar et al. 2013). Their role is underscored by the accumulation of mutations in additional regulators of their activation during the course of this tumor's treatment: MAPK (SPRY3) (Cabrita and Christofori 2008), PI3K (DEPDC5) (Cabrita and Christofori 2008; BarPeled et al. 2013), and WNT (KREMEN2, NET1, GPR124) (Orlow et al. 1987; Mao et al. 2002; Posokhova et al. 2015; Wei et al. 2017).

\section{Drivers of Recurrence and Susceptibility}

Although more cases will be needed to confidently identify the specific mutations that drive the expansion of therapy resistant subclones, the Hippo pathway stands out as a compelling potential driver of the fourth recurrence, induced by new mutations in LATS1 (missense) and in NF2 (nonsense). NF2 loss-of-function mutations have been previously associated with spinal ependymomas (Lee et al. 2016a), and recent evidence suggests that YAP1, the nuclear target of Hippo signaling, mediates aberrant proliferation upon NF2 loss during tumorigenesis (Shi et al. 2016). Furthermore, oncogenic YAP1 activation occurs as a consequence of a loss in NF2-dependent inactivation of LATS1 (a key inhibitor of YAP1), and decreased LATS1 activity has also been associated with glioma progression (Ji et al. 2012; Oh et al. 2015; Shi et al. 2016). YAP1 fusions are a key characteristic of one subgroup of supratentorial ependymomas (Archer and Pomeroy 2015) and we hypothesize that these mutations may represent convergent evolution, producing similar phenotypic effects via a different mechanism.

Only one substantial subclone was clearly responsive to therapy (cluster 3 ) and it was eradicated after treatment of recurrence 2 with Avastin and lapatinib. Among the compelling target mediators of response or biomarkers of response is the mutation in GPR124. This orphan member of the adhesion $\mathrm{G}$ protein-coupled receptor family is required specifically for the development of the brain vasculature in a VEGF-dependent manner (Kuhnert et al. 2010; Cullen et al. 2011; Zhou and Nathans 2014), and GPR124 may be a biomarker of Avastin response (Wang et al. 2014). GPR124 activates canonical Wnt signaling, which, as described above, is normally directly inhibited by MEN1 and KREMEN2. The genes for both of these proteins were mutated in this tumor, suggesting a model for Avastin response that might involve enhanced activation of a VEGF-Wnt axis.

\section{Changes to the Neoantigen Landscape during Progression}

Overall, the number of expressed MHC Class I neoantigens that we predicted was low, as expected in a tumor with only 110 protein-altering mutations. The presence of a relatively high burden in the founding clone suggests that mechanisms of immune evasion were already present when the initial tumor presented and may explain why there was no relationship between neoantigen load and subclonal response in subsequent tumors. This is supported by the observation that expression markers of T-cell activation were low in all five tumors. 


\section{Origin of New Subclones in Later Recurrences}

Although we present evidence suggesting that radiation and temozolomide treatment increased the mutation burden of this tumor, new mutations continued to be observed after their use was discontinued. It is important to note that this is not necessarily indicative of a continued elevated mutation rate. Mutations first observed in the last two recurrences may have existed at very low frequencies in prior time points, and the observed mutation spectrum in the new subclones is consistent with damage from previous therapies. Our prior work with ultradeep sequencing suggests that many rare subclones often exist in a tumor (with neutral fitness) and only become detectable after their fitness increases, either via acquisition of one or more driver mutations or because the therapeutic regimen changes the environment (Griffith et al. 2015b; Uy et al. 2017).

\section{Future Directions}

These results suggest that radiation and chemotherapy contributed to the increasing complexity of this tumor by both adding to the mutational burden and expanding the subclonal architecture. Determining whether this natural history is generally true in ependymoma progression, and what impact therapy-induced tumor evolution has on outcome, is an important area of investigation with the potential to alter how we treat patients with both completely resected supratentorial ependymoma and other brain tumors that are treated with irradiation but frequently recur. In the largest published study of ependymoma outcome involving 282 patients, GTR was the only prognostic factor associated with increased survival (VeraBolanos et al. 2015). In that analysis, GTR and postsurgical radiation therapy were associated with a shorter progression-free survival than GTR alone. Data presented here raise the alarming hypothesis that time-to-progression was shortened because irradiation promoted tumor evolution.

These clinical observations together with the sequencing-based characterizations presented here suggest that under some circumstances, adjuvant therapy may not be providing a benefit, and indeed may hasten recurrence by promoting molecular diversification of the tumor. We propose that this phenomenon be studied prospectively. In particular, our data suggest that completely resected supratentorial ependymomas, and possibly other brain tumors, should be sequenced at the time of diagnosis and again if there is a recurrent tumor. Over time, this might reveal the genotypes for which radiation therapy eradicates the founding clone, resulting in a cure, and in those for which it does not, but instead contributes to evolving tumor complexity. Ultimately, it may be prudent to initially observe those patients with complete resections without additional therapy or to treat those patients whose tumors are likely to evolve in response to radiation therapy with targeted agents only as dictated by genomic analysis. Critically important to this effort will be the use of unbiased sequencing approaches like whole-exome or whole-genome sequencing rather than sequencing of targeted gene panels. Although identification of druggable targets is important, it may be equally important to construct more global models of tumor evolution.

Finally, it will be important to investigate further the utility of genomic characterization to inform therapeutic options in this disease type. Although not all of the variants we identified were "druggable" in the classical sense, a subset were found to comprise predicted high-affinity neoantigen targets that, ultimately, formed the basis of a polyvalent personalized vaccine, administered after recurrence 4 . Such cancer immunogenomics approaches to clinical care are only made possible through comprehensive genomic approaches to tumor characterization. Although the efficacy of these treatments awaits large-scale studies and clinical trials that are ongoing, our case highlights the potential to consider the pursuit of a personalized vaccine in extremely challenging settings of multiply recurrent disease such as the one herein, where few to no other options exist. 


\section{METHODS}

\section{DNA Sequencing}

DNA was isolated from fresh frozen sections of each tumor resection using the QIAGEN Dual Prep and evaluated for quality and concentration using established methods. DNA was isolated from PBMC after Ficoll-based isolation to provide a normal comparator and was evaluated for quality and concentration. Using $500 \mathrm{ng}$ input for all five tumors and the blood normal DNA, we generated two indexed whole-genome sequencing libraries by standard methods (Kapa Biosystems) for each sample. One library per sample was processed through exome hybrid capture using the IDT xGEN research exome capture reagent (Integrated DNA Technologies), quantitated and amplified postcapture using the manufacturer's protocol. Each of the corresponding WGS libraries was amplified by PCR, quantitated, and diluted as appropriate for Illumina sequencing. The final libraries for each sample (WGS + exome) were pooled to produce combined tumor and normal WGS- and exome-sequencing data in a specific proportion, yielding $\sim 10$-fold WGS and 1000 -fold exome coverage (Supplemental Table S1). The resulting library pools were loaded onto the HiSegX platform and sequenced using 150-bp paired end reads.

\section{Somatic Variant Analysis}

After index-based binning of the reads into WGS- and exome-derived tumor and normal data, sequence data were aligned to reference sequence build GRCh37-lite-build37 using BWA-mem (Li, H. arXiv:1303.3997 [q-bio.GN]) version 0.7.10 (params: -t 8::), then merged and deduplicated using Picard version 1.113 (https://broadinstitute.github.io/picard/). Somatic variants were called from the combined data using our Genome Modeling System (Griffith et al. 2015a) as follows.

SNVs were detected using the union of four callers: (1) SAMtools (Li et al. 2009) version r982 (params: mpileup -BuDs) intersected with Somatic Sniper (Larson et al. 2012) version 1.0.4 (params: -F vcf -G -L -q $1-\mathrm{Q}$ 15) and processed through false-positive filter v1 (params: -bam-readcount- version 0.4 -bam-readcount-min-base-quality 15 -min-mapping-quality 40 -min-somatic-score 40), (2) VarScan (Koboldt et al. 2012) version 2.3.6 filtered by varscanhigh-confidence filter version $\mathrm{v} 1$ and processed through false-positive filter $\mathrm{v} 1$ (params: -bam-readcount-version 0.4 -bam-readcount-min-base-quality 15), (3) Strelka (Saunders et al. 2012) version 1.0.11 (params: isSkipDepthFilters =0), and (4) Mutect (Cibulskis et al. 2013) v1.1.4.

Indels were detected using the union of three callers: (1) GATK (McKenna et al. 2010) somatic-indel version 5336; (2) VarScan version 2.3.6 filtered by varscan-high-confidenceindel version v1, and (3) Strelka version 1.0.11 (params: isSkipDepthFilters $=0$ ).

SNVs and Indels were further filtered by removing artifacts found in a panel of 905 normal exomes, removing sites that exceeded $0.1 \%$ frequency in the 1000 genomes or $\mathrm{NHLBI}$ exome-sequencing projects, and then using a Bayesian classifier (https://github. $\mathrm{com} / \mathrm{genome} / \mathrm{genome} / \mathrm{blob} / \mathrm{master} / \mathrm{lib} / \mathrm{perl} /$ Genome/Model/Tools/Validation/Identify Outliers.pm) and retaining variants classified as somatic with a binomial log-likelihood of at least 10.

For protein-coding mutation counts described in the results below, a variant was considered to be present in a sample if it appeared with at least three variant supporting reads and a VAF of $>2.5 \%$. As some sites had low or variable coverage, a variant was only considered to be completely cleared if it did not appear in any subsequent samples.

Copy-number aberrations were detected using bam-window (window-size 10,000) and copy-cat version 1.6.11 (params: -per-read-length -per-library) (https://github.com/ chrisamiller/copyCat). Uneven sequence coverage of the normal sample required us to 
COLD SPRING HARBOR Molecular Case Studies
Treatment-linked resistance in an ependymoma run copyCat in tumor-only mode, followed by manual review to differentiate somatic from germline copy-number events.

Putative structural variants were detected using the union of BreakDancer 1.4.5 (Chen et al. 2009) filtered by novo-realign and tigra-sv, and squaredancer 0.1 (https://github. com/genome/genome/blob/master/lib/perl/Genome/Model/Tools/Sv/SquareDancer.pl).

\section{RNA Sequencing}

Total RNA was concurrently isolated from each fresh frozen tumor resection (QIAGEN Dual Prep), and evaluated for quality and concentration using the Agilent Tapestation. RNA-seq libraries were constructed using the TruSeq Stranded RNAseq library kit (Illumina, Inc.) according to the manufacturer's protocol, quantitated and diluted for sequencing. Using the HiSeq 2500, we produced sequencing data from each RNA-seq library in a single flow cell lane by paired end 100 bp reads, yielding between 96 and 655 million reads per sample. The fourth recurrence sample was subjected to a capture step before sequencing, using the IDT xGEN research exome capture reagent. This sample yielded 856 million reads, with a much higher coding-region percentage (Supplemental Table S1).

\section{RNA-seq Analysis}

The resulting read data were aligned to the human reference with TopHat v2.0.8 (denovo mode, params: -library-type fr-firststrand -bowtie-version=2.1.0). Expression levels were calculated with Cufflinks v2.1.1 (params: -max-bundle-length 10000000 -max-bundle-frags 10000000) (Trapnell et al. 2012).

\section{Gene Fusions}

Gene fusions were detected from RNA and DNA using Integrate v0.2.0 (Zhang et al. 2015) with default parameters.

\section{Heterogeneity Analysis}

Using the high depth of coverage from combining exome and WGS data sets for these tumors, we characterized the heterogeneity of each tumor specimen and compared it to the others in the series. Here, copy-number-neutral variants and their attendant VAFs were clustered in five dimensions using the sciClone algorithm v1.1 (Miller et al. 2014) (parameters: minimumDepth $=300$, maximumClusters $=15$ ), followed by phylogeny reconstruction with clonEvol (Dang et al. 2017).

\section{Neoantigen Predictions}

Somatic mutations and RNA-seq data from tumors were input into our pVACSeq pipeline (Hundal et al. 2016). WGS data from the normal blood was used to identify the patient's HLA haplotypes for Class I, using Laminar (Warren et al. 2012). MHC Class I binding predictions were generated through pVACSeq using NetMHC v3.4, as well as five other algorithms from the Immune Epitope Database and Analysis resource (IEDB, iedb.org): netMHC, netmhccons, netmhcpan, pickpocket, smm, and smmpmbec. Predictions were retained if the median score had an ic50 < 500 and better binding of the mutant peptide than the wild type (fold-change $>1$ ). Results were then filtered to require expression of the mutant allele (FPKM > 1 and at least one variant-supporting read in the RNA). These combined data sets were used to identify neoantigenic peptide sequences in all five tumor samples, as illustrated in Figure 3C. 
COLD SPRING HARBOR Molecular Case Studies
Treatment-linked resistance in an ependymoma
Competing Interest Statement

The authors have declared no competing interest.

Received November 15, 2017; accepted in revised form December 26, 2017.

\section{Pathology Methods}

All the resection specimens (original and recurrences) were handled as regular surgical neuropathology cases. Although H\&E stain and Ki-67 immunostain were performed on all the specimens, glial fibrillary acidic protein and EMA were limited to the initial and 2014 resections.

\section{ADDITIONAL INFORMATION}

\section{Data Deposition and Access}

Sequence data are available at dbGaP, under accession id phs001461.v1.p1. The Germline mutation list is accessible via the same dbGaP study. The variant was deposited in ClinVar (http://www.ncbi.nlm.nih.gov/clinvar/) and can be found under accession numbers SCV000678435-SCV000678467.

\section{Ethics Statement}

The patient had written and parental consent and the study was approved under Washington University in St. Louis IRB ID no. 201102299.

\section{Acknowledgments}

We thank early analysis efforts on this project from Charles Lu. We also gratefully acknowledge the sample intake, project tracking, and data production teams at the McDonnell Genome Institute. Funding for this project was generously provided from the McDonnell Genome Institute endowment and from the Robert E. and Louise F. Dunn Distinguished Professorship endowment to Washington University School of Medicine. We gratefully acknowledge the patient and her family.

\section{Author Contributions}

C.A.M., R.S.F., J.B.R., and E.R.M. contributed to conceptualization; C.A.M. and T.L. did formal analysis/data curation; S.D., M.D.S., J.B.R., and G.P.D. contributed to resources; C.A.M., J.B.R., and E.R.M. wrote the original draft; C.A.M., S.D., T.L., R.S.F., M.D.S., G.P.D., J.B.R., and E.R.M. did review and editing; C.A.M. did visualization; C.A.M., J.B.R., and E.R.M. supervised the study; and E.R.M. acquired funding.

\section{REFERENCES}

Adamo A, Fiore D, De Martino F, Roscigno G, Affinito A, Donnarumma E, Puoti I, Ricci-Vitiani L, Pallini R, Quintavalle $C$, et al. 2017. RYK promotes the stemness of glioblastoma cells via the WNT/ $\beta$-catenin pathway. Oncotarget 8: 13476-13487.

Al-Salameh A, François P, Giraud S, Calender A, Bergemer-Fouquet A-M, de Calan L, Goudet P, Lecomte P. 2010. Intracranial ependymoma associated with multiple endocrine neoplasia type 1. J Endocrinol Invest 33: 353-356.

Archer TC, Pomeroy SL. 2015. Defining the molecular landscape of ependymomas. Cancer Cell 27: 613-615. Bar-Peled L, Chantranupong L, Cherniack AD, Chen WW, Ottina KA, Grabiner BC, Spear ED, Carter SL, Meyerson M, Sabatini DM. 2013. A tumor suppressor complex with GAP activity for the Rag GTPases that signal amino acid sufficiency to mTORC1. Science 340: 1100-1106.

Behjati S, Gundem G, Wedge DC, Roberts ND, Tarpey PS, Cooke SL, Van Loo P, Alexandrov LB, Ramakrishna M, Davies $\mathrm{H}$, et al. 2016. Mutational signatures of ionizing radiation in second malignancies. Nat Commun 7: 12605.

Bocangel DB, Finkelstein S, Schold SC, Bhakat KK, Mitra S, Kokkinakis DM. 2002. Multifaceted resistance of gliomas to temozolomide. Clin Cancer Res 8: 2725-2734. 
Bodell WJ, Gaikwad NW, Miller D, Berger MS. 2003. Formation of DNA adducts and induction of lacl mutations in Big Blue Rat-2 cells treated with temozolomide: implications for the treatment of low-grade adult and pediatric brain tumors. Cancer Epidemiol Biomarkers Prev 12: 545-551.

Boersma V, Moatti N, Segura-Bayona S, Peuscher MH, van der Torre J, Wevers BA, Orthwein A, Durocher D, Jacobs JJL. 2015. MAD2L2 controls DNA repair at telomeres and DNA breaks by inhibiting $5^{\prime}$ end resection. Nature 521: 537-540.

Cabrita MA, Christofori G. 2008. Sprouty proteins, masterminds of receptor tyrosine kinase signaling. Angiogenesis 11: 53-62.

Cao Y, Liu R, Jiang X, Lu J, Jiang J, Zhang C, Li X, Ning G. 2009. Nuclear-cytoplasmic shuttling of menin regulates nuclear translocation of $\beta$-catenin. Mol Cell Biol 29: 5477-5487.

Chen K, Wallis JW, McLellan MD, Larson DE, Kalicki JM, Pohl CS, McGrath SD, Wendl MC, Zhang Q, Locke DP, et al. 2009. BreakDancer: an algorithm for high-resolution mapping of genomic structural variation. Nat Methods 6: 677-681.

Cheng P, Wang J, Waghmare I, Sartini S, Coviello V, Zhang Z, Kim S-H, Mohyeldin A, Pavlyukov MS, Minata M, et al. 2016. FOXD1-ALDH1A3 signaling is a determinant for the self-renewal and tumorigenicity of mesenchymal glioma stem cells. Cancer Res 76: 7219-7230.

Chung HJ, Choi YE, Kim ES, Han YH, Park MJ, Bae IH. 2015. miR-29b attenuates tumorigenicity and stemness maintenance in human glioblastoma multiforme by directly targeting BCL2L2. Oncotarget 6: 18429-18444

Cibulskis K, Lawrence MS, Carter SL, Sivachenko A, Jaffe D, Sougnez C, Gabriel S, Meyerson M, Lander ES, Getz G. 2013. Sensitive detection of somatic point mutations in impure and heterogeneous cancer samples. Nat Biotechnol 31: 213-219.

Cullen M, Elzarrad MK, Seaman S, Zudaire E, Stevens J, Yang MY, Li X, Chaudhary A, Xu L, Hilton MB, et al. 2011. GPR124, an orphan G protein-coupled receptor, is required for CNS-specific vascularization and establishment of the blood-brain barrier. Proc Natl Acad Sci 108: 5759-5764.

Dahlin AM, Hollegaard MV, Wibom C, Andersson U, Hougaard DM, Deltour I, Hjalmars U, Melin B. 2015. CCND2, CTNNB1, DDX3X, GLI2, SMARCA4, MYC, MYCN, PTCH1, TP53, and MLL2 gene variants and risk of childhood medulloblastoma. J Neurooncol 125: 75-78.

Dang HX, White BS, Foltz SM, Miller CA, Luo J, Fields RC, Maher CA. 2017. ClonEvol: clonal ordering and visualization in cancer sequencing. Ann Oncol. 28: 3076-3082.

Das M. 2016. MNX1: a novel prostate cancer oncogene. Lancet Oncol 17: e521.

Dorfer C, Tonn J, Rutka JT. 2016. Ependymoma: a heterogeneous tumor of uncertain origin and limited therapeutic options. Handb Clin Neurol 134: 417-431.

Funayama T, Sakane M, Yoshizawa T, Takeuchi Y, Ochiai N. 2013. Tanycytic ependymoma of the filum terminale associated with multiple endocrine neoplasia type 1: first reported case. Spine J 13: e49-e54.

Gallo A, Cuozzo C, Esposito I, Maggiolini M, Bonofiglio D, Vivacqua A, Garramone M, Weiss C, Bohmann D, Musti AM. 2002. Menin uncouples Elk-1, JunD and c-Jun phosphorylation from MAP kinase activation. Oncogene 21: 6434-6445.

Gao YF, Zhu T, Mao XY, Mao CX, Li L, Yin JY, Zhou HH, Liu ZQ. 2017. Silencing of Forkhead box D1 inhibits proliferation and migration in glioma cells. Oncol Rep 37: 1196-1202.

Griffith M, Griffith OL, Smith SM, Ramu A, Callaway MB, Brummett AM, Kiwala MJ, Coffman AC, Regier AA, Oberkfell BJ, et al. 2015a. Genome modeling system: a knowledge management platform for genomics. PLoS Comput Biol 11: e1004274.

Griffith M, Miller CA, Griffith OL, Krysiak K, Skidmore ZL, Ramu A, Walker JR, Dang HX, Trani L, Larson DE, et al. 2015b. Optimizing cancer genome sequencing and analysis. Cell Syst 1: 210-223.

Gurung B, Feng Z, Iwamoto DV, Thiel A, Jin G, Fan CM, Ng JM, Curran T, Hua X. 2013. Menin epigenetically represses Hedgehog signaling in MEN1 tumor syndrome. Cancer Res 73: 2650-2658.

Hegi ME, Diserens AC, Gorlia T, Hamou MF, de Tribolet N, Weller M, Kros JM, Hainfellner JA, Mason W, Mariani $L$, et al. 2005. MGMT gene silencing and benefit from temozolomide in glioblastoma. $N$ Engl $J$ Med 352: 997-1003.

Hundal J, Carreno BM, Petti AA, Linette GP, Griffith OL, Mardis ER, Griffith M. 2016. pVAC-Seq: a genomeguided in silico approach to identifying tumor neoantigens. Genome Med 8: 11.

Ji T, Liu D, Shao W, Yang W, Wu H, Bian X. 2012. Decreased expression of LATS1 is correlated with the progression and prognosis of glioma. J Exp Clin Cancer Res 31: 67.

Juhlin CC, Kiss NB, Villablanca A, Haglund F, Nordenström J, Höög A, Larsson C. 2010. Frequent promoter hypermethylation of the APC and RASSF1A tumour suppressors in parathyroid tumours. PLoS One 5: e9472.

Karnik SK, Hughes CM, Gu X, Rozenblatt-Rosen O, McLean GW, Xiong Y, Meyerson M, Kim SK. 2005. Menin regulates pancreatic islet growth by promoting histone methylation and expression of genes encoding p27Kip1 and p18INK4c. Proc Natl Acad Sci 102: 14659-14664.

Kato H, Uchimura I, Morohoshi M, Fujisawa K, Kobayashi Y, Numano F, Goseki N, Endo M, Tamura A, Nagashima C. 1996. Multiple endocrine neoplasia type 1 associated with spinal ependymoma. Intern Med 35: 285-289. 
Khatua S, Ramaswamy V, Bouffet E. 2017. Current therapy and the evolving molecular landscape of paediatric ependymoma. Eur J Cancer 70: 34-41.

Koboldt DC, Zhang Q, Larson DE, Shen D, McLellan MD, Lin L, Miller CA, Mardis ER, Ding L, Wilson RK. 2012. VarScan 2: somatic mutation and copy number alteration discovery in cancer by exome sequencing. Genome Res 22: 568-576.

Koga M, Matsuda M, Kawamura T, Sogo T, Shigeno A, Nishida E, Ebisuya M. 2014. Foxd1 is a mediator and indicator of the cell reprogramming process. Nat Commun 5: 3197.

Kuhnert F, Mancuso MR, Shamloo A, Wang HT, Choksi V, Florek M, Su H, Fruttiger M, Young WL, Heilshorn SC, et al. 2010. Essential regulation of CNS angiogenesis by the orphan G protein-coupled receptor GPR124. Science 330: 985-989.

Larson DE, Harris CC, Chen K, Koboldt DC, Abbott TE, Dooling DJ, Ley TJ, Mardis ER, Wilson RK, Ding L. 2012. SomaticSniper: identification of somatic point mutations in whole genome sequencing data. Bioinformatics 28: 311-317.

Lee $\mathrm{CH}$, Chung CK, Kim CH. 2016a. Genetic differences on intracranial versus spinal cord ependymal tumors: a meta-analysis of genetic researches. Eur Spine J 25: 3942-3951.

Lee $\mathrm{CH}$, Chung $\mathrm{CK}$, Ohn JH, Kim CH. 2016b. The similarities and differences between intracranial and spinal ependymomas : review from a genetic research perspective. J Korean Neurosurg Soc 59: 83-90.

Li H, Handsaker B, Wysoker A, Fennell T, Ruan J, Homer N, Marth G, Abecasis G, Durbin R. 2009. The sequence alignment/map format and SAMtools. Bioinformatics 25: 2078-2079.

Lindberg D, Akerström G, Westin G. 2008. Evaluation of CDKN2C/p18, CDKN1B/p27 and CDKN2B/p15 mRNA expression, and $\mathrm{CpG}$ methylation status in sporadic and MEN1-associated pancreatic endocrine tumours. Clin Endocrinol (Oxf) 68: 271-277.

Mabuchi M, Kataoka H, Miura Y, Kim T-S, Kawaguchi M, Ebi M, Tanaka M, Mori Y, Kubota E, Mizushima T, et al. 2010. Tumor suppressor, AT motif binding factor 1 (ATBF1), translocates to the nucleus with runt domain transcription factor 3 (RUNX3) in response to TGF- $\beta$ signal transduction. Biochem Biophys Res Commun 398: 321-325.

Mack SC, Witt H, Piro RM, Gu L, Zuyderduyn S, Stütz AM, Wang X, Gallo M, Garzia L, Zayne K, et al. 2014. Epigenomic alterations define lethal CIMP-positive ependymomas of infancy. Nature 506: 445-450.

Mao B, Wu W, Davidson G, Marhold J, Li M, Mechler BM, Delius H, Hoppe D, Stannek P, Walter C, et al. 2002. Kremen proteins are Dickkopf receptors that regulate Wnt/ $\beta$-catenin signalling. Nature 417: 664-667.

Matkar S, Thiel A, Hua X. 2013. Menin: a scaffold protein that controls gene expression and cell signaling Trends Biochem Sci 38: 394-402.

McGuire CS, Sainani KL, Fisher PG. 2009a. Both location and age predict survival in ependymoma: a SEER study. Pediatr Blood Cancer 52: 65-69.

McGuire CS, Sainani KL, Fisher PG. 2009b. Incidence patterns for ependymoma: a surveillance, epidemiology, and end results study. J Neurosurg 110: 725-729.

McKenna A, Hanna M, Banks E, Sivachenko A, Cibulskis K, Kernytsky A, Garimella K, Altshuler D, Gabriel S, Daly M, et al. 2010. The Genome Analysis Toolkit: a MapReduce framework for analyzing next-generation DNA sequencing data. Genome Res 20: 1297-1303.

Meng Z, Moroishi T, Mottier-Pavie V, Plouffe SW, Hansen CG, Hong AW, Park HW, Mo JS, Lu W, Lu S, et al. 2015. MAP4K family kinases act in parallel to MST1/2 to activate LATS1/2 in the Hippo pathway. Nat Commun 6: 8357.

Miller CA, White BS, Dees ND, Griffith M, Welch JS, Griffith OL, Vij R, Tomasson MH, Graubert TA, Walter MJ, et al. 2014. SciClone: inferring clonal architecture and tracking the spatial and temporal patterns of tumor evolution. PLoS Comput Biol 10: e1003665.

Oh JE, Ohta T, Satomi K, Foll M, Durand G, McKay J, Le Calvez-Kelm F, Mittelbronn M, Brokinkel B, Paulus W, et al. 2015. Alterations in the NF2/LATS1/LATS2/YAP pathway in Schwannomas. J Neuropathol Exp Neurol 74: 952-959.

Orlow S, Yasunami R, Boitard C, Bach JF. 1987. [Early induction of diabetes in NOD mice by streptozotocin]. CR Acad Sci III 304: 77-78.

Pajtler KW, Witt H, Sill M, Jones DTW, Hovestadt V, Kratochwil F, Wani K, Tatevossian R, Punchihewa C, Johann $P$, et al. 2015. Molecular classification of ependymal tumors across all CNS compartments, histopathological grades, and age groups. Cancer Cell 27: 728-743.

Pajtler KW, Mack SC, Ramaswamy V, Smith CA, Witt H, Smith A, Hansford JR, von Hoff K, Wright KD, Hwang E, et al. 2017. The current consensus on the clinical management of intracranial ependymoma and its distinct molecular variants. Acta Neuropathol 133: 5-12.

Posokhova E, Shukla A, Seaman S, Volate S, Hilton MB, Wu B, Morris H, Swing DA, Zhou M, Zudaire E, et al. 2015. GPR124 functions as a WNT7-specific coactivator of canonical $\beta$-catenin signaling. Cell Rep 10: 123-130.

Rosenthal R, McGranahan N, Herrero J, Taylor BS, Swanton C. 2016. deconstructSigs: delineating mutational processes in single tumors distinguishes DNA repair deficiencies and patterns of carcinoma evolution. Genome Biol 17: 31. 
Saunders CT, Wong WS, Swamy S, Becq J, Murray LJ, Cheetham RK. 2012. Strelka: accurate somatic smallvariant calling from sequenced tumor-normal sample pairs. Bioinformatics 28: 1811-1817.

Scacheri PC, Davis S, Odom DT, Crawford GE, Perkins S, Halawi MJ, Agarwal SK, Marx SJ, Spiegel AM, Meltzer PS, et al. 2006. Genome-wide analysis of menin binding provides insights into MEN1 tumorigenesis. PLoS Genet 2: e51.

Shi Y, Bollam SR, White SM, Laughlin SZ, Graham GT, Wadhwa M, Chen H, Nguyen C, Vitte J, Giovannini M, et al. 2016. Rac1-mediated DNA damage and inflammation promote Nf2 tumorigenesis but also limit cellcycle progression. Dev Cell 39: 452-465.

Trapnell C, Roberts A, Goff L, Pertea G, Kim D, Kelley DR, Pimentel H, Salzberg SL, Rinn JL, Pachter L. 2012. Differential gene and transcript expression analysis of RNA-seq experiments with TopHat and Cufflinks. Nat Protoc 7: 562-578.

Urioste M, Martínez-Ramírez A, Cigudosa JC, Colmenero I, Madero L, Robledo M, Martínez-Delgado B, Benítez J. 2002. Complex cytogenetic abnormalities including telomeric associations and MEN1 mutation in a pediatric ependymoma. Cancer Genet Cytogenet 138: 107-110.

Uy GL, Duncavage EJ, Chang GS, Jacoby MA, Miller CA, Shao J, Heath S, Elliott K, Reineck T, Fulton RS, et al. 2017. Dynamic changes in the clonal structure of MDS and AML in response to epigenetic therapy. Leukemia 31: 872-881.

Vera-Bolanos E, Aldape K, Yuan Y, Wu J, Wani K, Necesito-Reyes MJ, Colman H, Dhall G, Lieberman FS, Metellus $P$, et al. 2015. Clinical course and progression-free survival of adult intracranial and spinal ependymoma patients. Neuro Oncol 17: 440-447.

Wang Y, Cho SG, Wu X, Siwko S, Liu M. 2014. G-protein coupled receptor 124 (GPR124) in endothelial cells regulates vascular endothelial growth factor (VEGF)-induced tumor angiogenesis. Curr Mol Med 14: 543-554.

Wang Y, Ozawa A, Zaman S, Prasad NB, Chandrasekharappa SC, Agarwal SK, Marx SJ. 2011. The tumor suppressor protein menin inhibits AKT activation by regulating its cellular localization. Cancer Res 71: 371-382.

Warren RL, Choe G, Freeman DJ, Castellarin M, Munro S, Moore R, Holt RA. 2012. Derivation of HLA types from shotgun sequence datasets. Genome Med 4: 95.

Wei S, Dai M, Liu Z, Ma Y, Shang H, Cao Y, Wang Q. 2017. The guanine nucleotide exchange factor Net1 facilitates the specification of dorsal cell fates in zebrafish embryos by promoting maternal $\beta$-catenin activation. Cell Res 27: 202-225.

Wu Y, Feng ZJ, Gao SB, Matkar S, Xu B, Duan HB, Lin X, Li SH, Hua X, Jin G-H. 2012. Interplay between menin and K-Ras in regulating lung adenocarcinoma. J Biol Chem 287: 40003-40011.

Xu G, Chapman JR, Brandsma I, Yuan J, Mistrik M, Bouwman P, Bartkova J, Gogola E, Warmerdam D, Barazas M, et al. 2015. REV7 counteracts DNA double-strand break resection and affects PARP inhibition. Nature 521: 541-544.

Yuan Z, Sánchez Claros C, Suzuki M, Maggi EC, Kaner JD, Kinstlinger N, Gorecka J, Quinn TJ, Geha R, Corn A et al. 2016. Loss of MEN1 activates DNMT1 implicating DNA hypermethylation as a driver of MEN1 tumorigenesis. Oncotarget 7: 12633-12650.

Zhang J, White NM, Schmidt HK, Fulton RS, Tomlinson C, Warren WC, Wilson R, Maher CA. 2015. INTEGRATE: gene fusion discovery using whole genome and transcriptome data. Genome Res 26: 108-118.

Zhou Y, Nathans J. 2014. Gpr124 controls CNS angiogenesis and blood-brain barrier integrity by promoting ligand-specific canonical wnt signaling. Dev Cell 31: 248-256. 


\section{COLD SPRING HARBOR Molecular Case Studies}

\section{Resistance-promoting effects of ependymoma treatment revealed through genomic analysis of multiple recurrences in a single patient}

Christopher A. Miller, Sonika Dahiya, Tiandao Li, et al.

Cold Spring Harb Mol Case Stud 2018, 4: a002444 originally published online February 13, 2018 Access the most recent version at doi: $10.1101 / \mathrm{mcs} . a 002444$
Supplementary http://molecularcasestudies.cshlp.org/content/suppl/2018/02/13/mcs.a002444.D Material C1
References This article cites 71 articles, 13 of which can be accessed free at: http://molecularcasestudies.cshlp.org/content/4/2/a002444.full.html\#ref-list-1
License This article is distributed under the terms of the Creative Commons Attribution License, which permits unrestricted reuse and redistribution provided that the original author and source are credited.
Email Alerting Receive free email alerts when new articles cite this article - sign up in the box at the Service top right corner of the article or click here.

